\section{The neural networks of inhibitory control in post-traumatic stress disorder}

\section{EM Falconer ${ }^{1,2}$, R Bryant $^{1,2}, K_{\text {Felmingham }}{ }^{1}$, AH Kemp', G Olivieri' ${ }^{3}$, A Peduto ${ }^{3}$, E Gordon ${ }^{4}$, LM Williams ${ }^{1}$}

'The Brain Dynamics Centre, Westmead Millennium Institute, Westmead Hospital and Western Clinical School, University of Sydney, Australia; ${ }^{2}$ School of Psychology, University of New South Wales; ${ }^{3}$ Department of Radiology, Westmead Hospital; and ${ }^{4}$ The Brain Resource International Database, Brain Resource Company, Sydney, Australia

Post-traumatic stress disorder (PTSD) is associated with information-processing deficits and a reduction of selective attention, along with hyperarousal and hyperreactivity to threat- and emotion-related stimuli. PTSD may involve an enhancement in automatic processing and an inability to inhibit automatic processing when required. The predominant neurophysiological model of PTSD has focused on medial prefrontal disruptions during the processing of fear-related stimuli and does not include potential alterations in the explicit inhibitory control of automatic responding. We investigated executive inhibitory control in PTSD during an emotionally neutral (go/no go) response inhibition task in individuals with PTSD $(n=23)$ and matched healthy controls $(n=23)$ using functional magnetic resonance imaging. PTSD was associated with diminished inhibitory control and reduced activation of a cortical inhibitory network (particularly right ventrolateral prefrontal cortex), as well as an increase in areas associated with sensory processing (somatosensory and visual cortices) and increased inhibitory task demand (striatum). These findings are consistent with diminished executive inhibitory control in PTSD and may reflect increased stimulus processing, which undermines cortical control mechanisms.

\section{4-04}

\section{Treatment response and post-traumatic stress disorder: neuroimaging findings}

\section{RA Bryantt ${ }^{1,2}, \mathrm{KL}^{2}$ Felmingham ${ }^{1,3}$, EM Falconer $^{1,2}$, AH Kemp ${ }^{1,3}$, P Das ${ }^{1,4}$, A Peduto ${ }^{1,5}$, LM Williams ${ }^{1,3}$}

'The Brain Dynamics Centre, Westmead Millennium Institute, Westmead Hospital and Western Clinical School, University of Sydney, Australia; ${ }^{2}$ School of Psychology, University of New South Wales; ${ }^{3}$ Psychological Medicine, Western Clinical School, University of Sydney; ${ }^{4}$ Neuroscience Institute of Schizophrenia and Allied Disorders (NISAD), New South Wales, Australia; and 5Department of Radiology, Westmead Hospital, Westmead, Australia
Biological models propose that post-traumatic stress disorder (PTSD) reflects a failure of extinction of the conditioned fear response. Animal models and recent human imaging studies suggest that ventromedial prefrontal (vmPFC) regions inhibit amygdala fear networks during fear extinction (Rauch et al. 2006; Phelps et al. 2004). Accordingly, PTSD is associated with a failure of vmPFC activity in response to threat. Exposure-based treatments are thought to facilitate the extinction of conditioned fear. No imaging studies have examined the neural correlates of symptom improvement following exposure-based treatment in PTSD. Eight individuals with PTSD underwent functional magnetic resonance imaging (fMRI) scanning while viewing fearful and neutral facial expressions in a passive viewing task adapted to a $1.5 \mathrm{~T}$ scanner. fMRI assessments were conducted before and after treatment. Amygdala and vmPFC (anterior cingulate) activity was examined before and after treatment in a repeated-measures, fixed-effects ANOVA and changes in these regions were correlated with changes in PTSD severity. Consistent with predictions, findings show that anterior cingulate activity increased and amygdala activity reduced to fear following exposure treatment in PTSD, and symptom improvement was correlated with increased anterior cingulate activity and reduced amygdala activity to fear. These findings provide initial support for a role of reduced vmPFC activity in PTSD that recovers following exposure treatment.

\section{4-05}

\section{Neural activity in dissociative and nondissociative PTSD: an fMRI analysis of conscious and nonconscious fear processing}

\section{KL Felmingham ${ }^{1,2}$, LM Williams ${ }^{1,2}$, E Falconer ${ }^{1,3}$, AH Kemp ${ }^{1,2}$, P Das ${ }^{1,4}$, A Peduto ${ }^{1,5}$, RA Bryant ${ }^{1,3}$}

\footnotetext{
'The Brain Dynamics Centre, Westmead Millennium Institute, Westmead Hospital and Western Clinical School, University of Sydney, Australia; 'Psychological Medicine, Western Clinical School, University of Sydney; School of Psychology, University of New South Wales; ${ }^{3}$ Neuroscience Institute of Schizophrenia and Allied Disorders (NISAD), New South Wales, Australia; and ${ }^{4}$ Department of Radiology, Westmead Hospital, Westmead, Australia
}

Post-traumatic stress disorder (PTSD) involves variable responses to threat, ranging from hyperreactive to dissociative. While most imaging research has explored hyperreactivity in PTSD, an important but poorly understood subtype of PTSD is dissociation. Recent imaging research suggests that dissociative 
PTSD is associated with increased medial prefrontal activity in response to threat (Lanius et al. 2002). This accords with neurobiological models that implicate a corticolimbic disconnection in dissociation (Sierra \& Berrios 1998). It is critical to explore both the cortical and subcortical networks associated with dissociation during different levels of awareness of fear processing in PTSD. We predicted that whereas conscious processing of fear would be associated with increased mPFC activity, nonconscious processing would bypass inhibitory control and be associated with exaggerated amygdale activity. Twenty-three participants with PTSD, classified as dissociative $(n=12)$ or nondissociative $(n=11)$, viewed masked and unmasked fearful and neutral facial expressions. Amygdala and anterior cingulate function was examined with functional magnetic resonance imaging. In line with our predictions, the dissociative PTSD group showed increased ventral anterior cingulate activity to conscious fear faces relative to nondissociative PTSD. In contrast, the dissociative group showed bilateral amygdala activity to masked fear faces compared with nondissociative PTSD. These findings suggest that dissociative PTSD is associated with enhanced automatic amygdala activity under masked conditions and increased regulatory prefrontal processing under controlled processing conditions.

\section{Schizophrenia and Bipolar Disorder in the Real World - 'Best Buys and Best Bets'}

\section{J Kulkarni', P McGorry ${ }^{2}$}

'Alfred Psychiatry Research Centre, The Alfred, Prahran, Victoria, Australia; and ${ }^{2}$ ORYGEN Research Centre, ORYGEN Youth Health, Parkville, Victoria, Australia

\section{Overview}

Given the high cost of serious mental illness, both in terms of cost to the heath care system and to the person and the society in which they live, it is important that considerable attention be given to the policies and health services that manage these conditions. We present a series of 'real-world' studies evaluating the clinical and economic outcomes of disorders such as schizophrenia and bipolar disorder, including early-intervention models and their evaluation, which are necessary to help determine where to invest scarce resources in the Australian mental health care system.

\section{The Australian SCAP Study: real- world schizophrenia - outcomes and economics}

\section{J Kulkarni', P Fitzgerald', AR Castella', K Filia', S Filia', D Jackson' ${ }^{2}$, L Christova², W Montgomery ${ }^{3}$}

'Alfred Psychiatry Research Centre, The Alfred and Monash University Department of Psychological Medicine, Victoria, Australia; ${ }^{2}$ M-TAG Australia; and

${ }^{3}$ Eli Lilly Pty Ltd, Australia

Background: In a climate of intense debate about where to invest scarce resources in Australia's mental health treatment system, to achieve optimal outcomes, 'real-world' studies, such as the Schizophrenia Care and Assessment Program, provide comprehensive and broad-ranging data that undermine 'hypothetically' based models that deter investment in low-prevalence disorders such as schizophrenia.

Methods: A cohort of 347 patients with schizophrenia was followed up over 3 years. Clinical outcomes were assessed at 6 monthly intervals, and resource utilization and costing data was collected continuously from internal and external databases as well as from participants directly.

Results: The majority of participants showed significant improvement in clinical outcome measures of symptomatology, functioning and quality of life, although the level of employment remained low throughout the study. A small minority of participants consumed a disproportionate amount of resources and costs. Despite an increase in the use of the more expensive atypical antipsychotic medications, the total treatment costs for the cohort decreased significantly over the course of the study, largely related to a reduction in hospitalizations.

Conclusions: For the majority of people with schizophrenia, investment in assertive treatment programs and measures to reduce hospitalization will result in enhanced functioning and quality of life, as well as a reduction in long-term societal and government costs. Investment in employment rehabilitation programs is an important step to completing the positive gains made by people like those who participated in the Australian SCAP. 